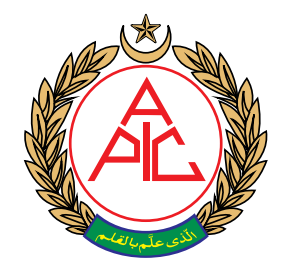

\title{
Oxygen therapy
}

\author{
Mayuri Gupta $^{1}$, Pranav Bansal ${ }^{2}$, Shivani Sharma ${ }^{3}$ \\ ${ }^{1}$ Resident; ${ }^{2}$ Professor \& Head, Department of Anesthesiology \& Critical Care, BPS \\ Govt Medical College, Khanpur Kalan, Sonipat, Haryana, India. \\ ${ }^{3}$ Professor, Dept of Physiology, Medical University of the Americas, St. Kitts \& \\ Nevis, USA.
}

Citation: Gupta M, Bansal P, Sharma S. Oxygen therapy (Cliniquiz). Anaesth. pain intensive care. 2020;24(2):249-250

DOI: https://doi.org/10.35975/apic.v24i2.

A 55-year old male patient with history of smoking for the past 30-years presents to emergency room with complains of respiratory distress since 2-days. On examination his respiratory rate is $30 /$ min with accessory muscles of respiration at work. His $\mathrm{SpO}_{2}$ is $82 \%$ on room air. $\mathrm{He}$ is administered $\mathrm{O}_{2}$ via face mask with reservoir bag @ $5 \mathrm{~L} / \mathrm{min}$ and improvement in $\mathrm{SpO}_{2}$ observed is $90 \%$. His is therefore administered a trial of BIPAP and his condition stabilizes over next few hours

(Please select the one best answer)

Q 1. Which of the following is not an absolute indication for oxygen therapy:-
A. Severe hypoxemia
B. Severe hypercapnia
C. Carbon monoxide poisoning
D. Shock

Q 2. Which of the following is not an example of low flow variable performance devices?
A. Nasal cannula
B. Hudson's mask
C. Oxygen blender
D. Non- rebreather face mask

Q 3. The maximum oxygen flow recommended through nasal cannula is:-
A. $2 \mathrm{~L} / \mathrm{min}$
B. $4 \mathrm{~L} / \mathrm{min}$
C. $6 \mathrm{~L} / \mathrm{min}$
D. $8 \mathrm{~L} / \mathrm{min}$

Q 4. Which of the following statement is true about NO DESAT (Nasal Oxygenation During Efforts Securing A Tube)?

A. It is a simple method of preoxygenation

B. Hot and humidified oxygen is supplied through nasal cannula
C. It increases risk of barotrauma due to higher flow

D. Rate of oxygen flow is $5-15 \mathrm{~L} / \mathrm{min}$

Q 5. Which of the following are approximate values of oxygen consumption/min and carbon dioxide elimination/min in a healthy adult?
A. $250 \mathrm{ml}$ and $200 \mathrm{ml}$ respectively
B. $500 \mathrm{ml}$ and $450 \mathrm{ml}$ respectively
C. $200 \mathrm{ml}$ and $250 \mathrm{ml}$ respectively
D. $450 \mathrm{ml}$ and $500 \mathrm{ml}$ respectively

Q 6. Maximum fraction of oxygen that can be delivered via Venturi mask is:-
A. $40 \%$
B. $50 \%$
C. $60 \%$
D. $70 \%$

Q 7. Which of the following is an advantage of preoxygenation?
A. Prolongation of duration of apnoea to 5- 6 minutes
B. Early detection of esophageal intubation
C. Prevention of absorption atelectasis
D. Production of reactive oxygen species 
Q 8: Which of the following is not true for THRIVE

A. It stands for Transnasal Humidified Rapid Intubation and Ventilatory Expansion

B. It provides high flow oxygen rates from $35-70 \mathrm{~L} / \mathrm{min}$

C. It supplies hot and humidified oxygen with positive pressure

D. The positive pressure prevents atelectasis

Q 9: What is the principle of gas entrainment in Venturi mask?
A. Coanda effect
B. Bernoulli's principle
C. Hagen Poiseuille's equation
D. Graham's law

Q 10: Which of the following is not an example of fixed performance oxygen delivery device?
A. Venturi mask
B. Oxygen blender
C. Non- rebreathing mask with reservoir bag
D. CPAP device

\section{ANSWERS}

A. 1(B): Common indications for oxygen therapy include Acute and chronic arterial hypoxemiA. Chronic type II respiratory failure, carbon monoxide poisoning and metabolic acidosis

A. 2(B): Low flow variable performance devices include Nasal prongs, Simple face mask/ Hudson's mask with or without reservoir bag, Non- rebreather mask and tracheostomy mask without entrainment device. High flow fixed performance device include Venturi mask, Tracheostomy mask and face mask with entrainment device, CPAP and BIPAP device.

A. 3(C): The range of oxygen flow recommended through nasal cannula is $2-6 \mathrm{~L} / \mathrm{min}$. Flows higher than $6 \mathrm{~L} / \mathrm{min}$ are uncomfortable to the patent and cause drying of nasal mucosa.
A. 4(D): NO DESAT (Nasal Oxygenation During Efforts Securing A Tube) is a simple method of paraoxygenation. The rate of oxygen flow is 5-15 $\mathrm{L} / \mathrm{min}$ and it is supplied through nasal cannula at the time of securing airway. This strategy improves oxygen reserves during apnea and delays the development of hypoxemia.

A. 5(A): The approximate rate of oxygen consumption in a healthy adult is $250-300 \mathrm{ml} / \mathrm{min}$ and carbon dioxide elimination is $200 \mathrm{ml} / \mathrm{min}$.

A. 6(C): Maximum fraction of oxygen that can be delivered through Venturi mask is $60 \%$

A. 7(C): Disadvantage of preoxygenation include absorption atelectasis, delayed detection of esophageal intubation and production of reactive oxygen species

A. 8(A): THRIVE stands for Transnasal Humidified Rapid Insufflation Ventilatory Exchange.

The high flow of oxygen provides a CPAP of 3-5 $\mathrm{cm} / \mathrm{H}_{2} \mathrm{O}$ that prevents atelectasis of alveoli and allows exchange of oxygen and carbon dioxide.

A. 9(B): Venturi effect is based on Bernoulli principle. It states that when velocity of the gas flowing through a narrow orifice increases, there is a pressure drop across the orifice. This pressure drop creates a negative suction pressure that causes entrainment of air.

A. 10(C) Fixed performance devices or high-flow systems provide a constant $\mathrm{FiO}_{2}$ by delivering the gas at flow rates that exceed the patient's peak inspiratory flow rate and by using devices that entrain a fixed proportion of room air. In contrast, low-flow systems provide oxygen at flow rates that are lower than patients' inspiratory demands. Here the final concentration of oxygen delivered depends on the ventilatory demands of the patient, the size of the oxygen reservoir, and the rate at which the reservoir is filled. At a low flow, the larger the tidal volume, the lower is the delivered $\mathrm{FiO}_{2}$ and vice versa. 